Herzschr Elektrophys 2019 · 30:377-388 https://doi.org/10.1007/s00399-019-00647-w Online publiziert: 8. November 2019

(c) Springer Medizin Verlag GmbH, ein Teil von Springer Nature 2019

\section{Inhaltsverzeichnis}

- Einleitung

- Datenbasis

- Datenvolumen

- Demografische Daten

- Implantationen

- EKG-Indikation zur Schrittmacherimplantation

- Auswahl der Schrittmachersysteme

- Operationsdaten

- Aggregatwechsel

- Revisionen/Systemwechsel/ Explantationen

- Kommentar mit internationalem

Vergleich

- Datenbasis

- EKG-Indikationen zur Schrittmachertherapie

- Schrittmachersystemauswahl

- Operationsdaten

- Zusammenfassung und Ausblick

- Literatur

- Tabellenverzeichnis

- Abbildungsverzeichnis

Die Mitglieder der Bundesfachgruppe Herzschrittmacher und Defibrillatoren werden am Beitragsende gelistet.

\author{
A. Markewitz ${ }^{1}$ Bundesfachgruppe Herzschrittmacher und Defibrillatoren \\ 'Bendorf, Deutschland
}

\title{
Jahresbericht 2017 des Deutschen Herzschrittmacher- und Defibrillator-Registers - Teil 1: Herzschrittmacher
}

\section{Fachgruppe Herzschrittmacher und Defibrillatoren beim IQTIG - Institut für Qualitätssicherung und Transparenz im Gesundheitswesen}

\section{Einleitung}

Der 17. Jahresbericht des Deutschen Herzschrittmacher- und DefibrillatorRegisters unterscheidet sich wie in den letzten beiden Jahren weiterhin von den vorangehenden Berichten seit 2002, weil die Details der sekundären Nutzung der bei den verpflichtenden Maßnahmen der Qualitätssicherung erhobenen Daten immer noch ungeklärt sind. Damit fehlen in diesem Bericht erneut viele der früher üblichen Auswertungen und die Tabellen des Anhangs, da die in diesem Bericht wiedergegebenen Daten ausschließlich wegen ihres primären Zwecks, der Qualitätssicherung, verfügbar sind, und die vielen sonstigen Daten, die ggf. nach Sonderauswertungen der Versorgungsforschung dienen könnten, bisher nicht genutzt werden dürfen. Damit ist dieser Bericht auch wieder deutlich kürzer als zuvor.

Grundlage des Berichts sind die Bundesauswertungen der für die jeweiligen Verfahren der externen Qualitätssicherung publizierten Daten [1], für einige Vergleiche wurden zudem die Daten vorausgegangener Berichte des Deutschen Herzschrittmacher- und Defibrillator-Registers verwendet [2].

Die im Folgenden vorgestellten und kommentierten Ergebnisse der Opera- tionen bei Patienten mit Herzschrittmachern (Teil 1) und implantierbaren Kardioverter-Defibrillatoren (ICD) (Teil 2) aus dem stationären Bereich im Jahr 2017 in Deutschland geben einen deutlich gröberen Überblick über die Situation bei kardialen Rhythmusimplantaten als zuvor. Der traditionelle Vergleich mit den Registern aus Schweden und der Schweiz ist dennoch möglich [3, 4]. Aus Dänemark gibt es seit 2016 keine neuen Berichte mehr.

Die Zahlen aus dem ambulanten Bereich fehlen weiterhin, sodass dieser Bericht nicht über alle in Deutschland durchgeführten Eingriffe bei Herzschrittmachern berichtet. Wie viele Operationen ambulant durchgeführt werden, entzieht sich unserer Kenntnis. Es ist davon auszugehen, dass bei den isolierten Aggregatwechseln ein relevanter Anteil der Eingriffe im ambulanten Sektor erbracht werden, zumal diese Eingriffe nur noch in Einzelfällen angemessen vergütet werden, wenn sie unter stationären Bedingungen durchgeführt werden.

\section{Datenbasis}

\section{Datenvolumen}

Die Anzahl der Institutionen, die Herzschrittmacheroperationen durchführen, 


\begin{tabular}{|c|c|c|c|}
\hline Datenbasis & 2015 & 2016 & 2017 \\
\hline \multicolumn{4}{|l|}{ Krankenhäuser } \\
\hline 09/1: Implantationen & 1128 & 1111 & 1102 \\
\hline 09/2: Aggregatwechsel & 985 & 957 & 957 \\
\hline 09/3: Revisionen/Systemwechsel/Explantationen & 946 & 925 & 923 \\
\hline \multicolumn{4}{|l|}{ Eingriffe } \\
\hline 09/1: Implantationen & 75.731 & 77.188 & 77.283 \\
\hline 09/2: Aggregatwechsel & 18.725 & 18.575 & 17.085 \\
\hline 09/3: Revisionen/Systemwechsel/Explantationen & 12.209 & 11.822 & 11.383 \\
\hline Alle 3 Leistungsbereiche (Summe) & 106.665 & 107.585 & 105.751 \\
\hline
\end{tabular}

Tab. 3 Vollzähligkeit der ausgewerteten Datensätze bzw. Krankenhäuser im Jahr 2016

\begin{tabular}{|c|c|c|c|}
\hline Datenbasis 2016 & Soll & Ist [1] & Vollzähligkeit (\%) \\
\hline \multicolumn{4}{|l|}{ Krankenhäuser } \\
\hline 09/1 Implantationen & 1117 & 1111 & 99,5 \\
\hline 09/2 Aggregatwechsel & 954 & 957 & 100,3 \\
\hline $\begin{array}{l}\text { 09/3 Revisionen/Systemwechsel/ } \\
\text { Explantationen }\end{array}$ & 927 & 925 & 99,8 \\
\hline \multicolumn{4}{|l|}{ Eingriffe } \\
\hline 09/1 Implantationen & 77.514 & 77.293 & 99,7 \\
\hline 09/2 Aggregatwechsel & 18.518 & 18.734 & 101,2 \\
\hline $\begin{array}{l}\text { 09/3 Revisionen/Systemwechsel/ } \\
\text { Explantationen }\end{array}$ & 11.891 & 11.879 & 99,9 \\
\hline
\end{tabular}

Tab. 4 Vollzähligkeit der ausgewerteten Datensätze bzw. Krankenhäuser im Jahr 2017

\begin{tabular}{|c|c|c|c|}
\hline Datenbasis 2017 & Soll & Ist [1] & Vollzähligkeit (\%) \\
\hline \multicolumn{4}{|l|}{ Krankenhäuser } \\
\hline 09/1 Implantationen & 1104 & 1102 & 99,8 \\
\hline 09/2 Aggregatwechsel & 950 & 957 & 100,7 \\
\hline $\begin{array}{l}\text { 09/3 Revisionen/Systemwechsel/ } \\
\text { Explantationen }\end{array}$ & 922 & 923 & 100,1 \\
\hline \multicolumn{4}{|l|}{ Eingriffe } \\
\hline 09/1 Implantationen & 77.363 & 77.370 & 100,0 \\
\hline 09/2 Aggregatwechsel & 16.952 & 17.089 & 100,8 \\
\hline $\begin{array}{l}09 / 3 \text { Revisionen/Systemwechsel/ } \\
\text { Explantationen }\end{array}$ & 11.420 & 11.421 & 100,0 \\
\hline
\end{tabular}

geht seit 2011 kontinuierlich zurück. Die Zahl der Neuimplantationen ist nahezu gleich geblieben, wohingegen die Zahlen für die Aggregatwechsel und Revisionen leicht abgenommen haben, sodass die Gesamtzahl der Schrittmacheroperationen um knapp 2000 Eingriffe abgenommen hat (- Tab. 1).

Die Zahl der Institutionen, die nur sehr gelegentlich Schrittmacheroperatio-
Tab. 2 Operationsvolumina (Implantatio-

nen, Aggregatwechsel und Revisionen) der

meldenden Krankenhäuser

\begin{tabular}{|c|c|c|c|}
\hline \multirow{2}{*}{$\begin{array}{l}\text { Anzahl } \\
\text { gemeldeter } \\
\text { Herzschritt- } \\
\text { macheropera- } \\
\text { tionen }\end{array}$} & \multicolumn{3}{|c|}{ Krankenhäuser } \\
\hline & 2015 & 2016 & 2017 \\
\hline \multicolumn{4}{|c|}{ Neuimplantationen } \\
\hline$<20$ & 313 & 295 & 293 \\
\hline 20 und mehr & 810 & 814 & 806 \\
\hline \multicolumn{4}{|l|}{ Aggregatwechsel } \\
\hline$<20$ & 627 & 624 & 650 \\
\hline 20 und mehr & 357 & 333 & 307 \\
\hline \multicolumn{4}{|c|}{ Revisionen/Systemwechsel/Explantationen } \\
\hline$<20$ & 776 & 774 & 772 \\
\hline 20 und mehr & 169 & 150 & 150 \\
\hline
\end{tabular}

\section{Demografische Daten}

Die Zahl der Eingriffe pro Institution hat nur bei den Neuimplantationen geringfügig zugenommen, ansonsten abgenommen, die Verweildauer wird inzwischen nicht mehr kürzer, und die Rate an permanent stimulationsbedürftigen, d.h. schrittmacherabhängigen Patienten wird nicht mehr ermittelt. Der Anteil an jungen Patienten ( $<60$ Jahre) nimmt ebenso wie der Anteil betagter Patienten (>90 Jahre) in allen Eingriffsklassen zu. Bei den übrigen Ergebnissen zeigen sich allenfalls marginale Veränderungen (- Tab. 5).

\section{Implantationen}

\section{EKG-Indikation zur Schrittmacher- implantation}

Bei den Indikationen zur Schrittmacherimplantation haben im Jahr 2017 die Indikationsgruppen AV-Block und die Indikationen zur CRT in Absolutzahlen zugenommen, alle anderen Indikationsgruppen haben abgenommen. Bei der relativen Verteilung ergeben sich nur marginale Verschiebungen (• Tab. 6).

Die Leitlinienkonformität ist im Jahr 2017 wie im Vorjahr vorausgesagt im Vergleich zu 2016 von 88,62\% auf $92,75 \%$ angestiegen und liegt damit wieder im 
Tab. 5 Demografische Daten zu Implantationen, Aggregatwechsel und Revisionen/Systemwechsel/Explantationen

\begin{tabular}{|c|c|c|c|c|}
\hline & & 2015 & 2016 & 2017 \\
\hline \multirow{7}{*}{$\begin{array}{l}09 / 1 \\
\text { Implanta- } \\
\text { tionen }\end{array}$} & Anzahl Eingriffe & 75.730 & 77.188 & 77.283 \\
\hline & - Im Mittel je Institution & 67,1 & 69,5 & 70,1 \\
\hline & Männliche Patienten & $55,6 \%$ & $56,0 \%$ & $56,7 \%$ \\
\hline & Weibliche Patienten & $44,4 \%$ & $44,0 \%$ & $43,3 \%$ \\
\hline & Patienten $<60$ Jahre & $6,0 \%$ & $6,0 \%$ & $6,2 \%$ \\
\hline & Patienten 90 Jahre und älter & $5,0 \%$ & $5,0 \%$ & $5,4 \%$ \\
\hline & Mittlere postoperative Verweildauer (Tage) & 4,7 & 4,6 & 4,6 \\
\hline \multirow{7}{*}{$\begin{array}{l}09 / 2 \\
\text { Aggregat- } \\
\text { wechsel }\end{array}$} & Anzahl Eingriffe & 18.725 & 18.575 & 17.085 \\
\hline & - Im Mittel je Institution & 19,0 & 19,4 & 17,9 \\
\hline & Männliche Patienten & $52,4 \%$ & $52,3 \%$ & $52,7 \%$ \\
\hline & Weibliche Patienten & $47,3 \%$ & $47,7 \%$ & $47,3 \%$ \\
\hline & Patienten $<60$ Jahre & $5,5 \%$ & $5,1 \%$ & $5,2 \%$ \\
\hline & Patienten 90 Jahre und älter & $12,3 \%$ & $13,2 \%$ & $13,3 \%$ \\
\hline & Mittlere postoperative Verweildauer (Tage) & 1,9 & 1,8 & 1,8 \\
\hline \multirow{7}{*}{$\begin{array}{l}09 / 3 \\
\text { Revisionen, } \\
\text { System- } \\
\text { wechsel, } \\
\text { Explanta- } \\
\text { tionen }\end{array}$} & Anzahl Eingriffe & 12.209 & 11.822 & 11.383 \\
\hline & - Im Mittel je Institution & 12,9 & 12,8 & 12,3 \\
\hline & Männliche Patienten & $54,5 \%$ & $55,0 \%$ & $55,0 \%$ \\
\hline & Weibliche Patienten & $45,5 \%$ & $45,0 \%$ & $45,0 \%$ \\
\hline & Patienten $<60$ Jahre & $9,7 \%$ & $10,2 \%$ & $10,8 \%$ \\
\hline & Patienten 90 Jahre und älter & $5,6 \%$ & $6,0 \%$ & $6,2 \%$ \\
\hline & Mittlere postoperative Verweildauer (Tage) & 4,7 & 4,9 & 4,9 \\
\hline
\end{tabular}

Tab. 6 Führende EKG-Indikationen zur Schrittmacherimplantation in Absolutzahlen sowie in Prozent im Vergleich zum Vorjahr

\begin{tabular}{|c|c|c|c|c|}
\hline \multirow[t]{2}{*}{ EKG-Befunde } & \multicolumn{2}{|l|}{2016} & \multicolumn{2}{|l|}{2017} \\
\hline & $n$ & $\%$ & $n$ & $\%$ \\
\hline AV-Block & 34.024 & 44,1 & 35.310 & 45,7 \\
\hline Sick-Sinus-Syndrom (SSS) ${ }^{a}$ & 27.972 & 36,2 & 26.981 & 34,9 \\
\hline Vorhofflimmern & 11.141 & 14,4 & 10.963 & 14,2 \\
\hline Schenkelblöcke & 918 & 1,2 & 843 & 1,1 \\
\hline Indikation zu CRT & 1588 & 2,1 & 1910 & 2,5 \\
\hline Sonstiges & 1545 & 2,0 & 1276 & 1,7 \\
\hline Summe & 77.188 & 100 & 77.283 & 100 \\
\hline
\end{tabular}

Tab. 7 Venöser Zugang bei Schrittmacherimplantationen 2017 im Vergleich zu den Vorjahren

\begin{tabular}{llllllll} 
Venöser Zugang & $\mathbf{2 0 1 5}$ & \multicolumn{3}{l}{$\mathbf{2 0 1 6}$} & \multicolumn{2}{c}{$\mathbf{2 0 1 7}$} \\
& $\boldsymbol{n}$ & $\mathbf{\%}$ & $\boldsymbol{n}$ & $\mathbf{\%}$ & $\boldsymbol{n}$ & $\%$ \\
\hline Vena cephalica & 29.385 & 38,8 & 30.464 & 39,5 & 30.796 & 39,9 \\
Vena subclavia & 49.786 & 65,7 & 49.630 & 64,3 & 49.232 & 63,7 \\
\hline Andere & 1312 & 1,7 & 1500 & 1,9 & 1697 & 2,2
\end{tabular}

grünen Bereich. Bezogen auf die einzelnen Indikationen liegt die Leitlinienkonformität für die AV-Blockierungen $\geq$ II. Grades und die Bradykardie mit permanentem Vorhofflimmern bei über $97 \%$, für alle anderen Rhythmusstörungen unter $90 \%$ [1]. Dies ist bei der zahlenmäßig größten Gruppe, den Sinusknotenerkrankungen, vermutlich mit der bei Patienten mit dieser Rhythmusstörung nicht selten schwierigen Korrelation von Symptomen und EKG-Befunden verursacht. Zudem sind Leitlinientexte nicht immer gut in messbare Parameter für die externe Qualitätssicherung umzusetzen, was in Summe erklären kann, warum die Indikationsstellung nicht bei allen Rhythmusstörungen die Erwartungen vollständig erfüllen konnte.

\section{Auswahl der Schrittmacher- systeme}

Die Auswahl der Schrittmachersysteme zeigt weiterhin, dass in der täglichen Praxis überwiegend VVI-Systeme oder DDD-Systeme implantiert werden (• Abb. 1). Dabei hat die Zahl der VVISysteme leicht abgenommen und die Zahl der CRT-Systeme mit Vorhofsonde leicht zugenommen.

Die Leitlinienkonformität bei der Systemauswahl liegt weiterhin bei über $98 \%$ [1].

Die Hersteller der verwendeten Aggregate werden seit 2017 ebenso wie die Polarität und die Fixationsmechanismen der verwendeten Sonden nicht mehr erfasst.

\section{Operationsdaten}

Nachdem die Ergebnisse beim venösen Zugang bis 2015 den gleichen Trend in die falsche Richtung zeigten, hat in 2017 die Verwendung der V. cephalica als venöser Zugang erneut zugenommen (• Tab. 7). Die Zahl der Institutionen, in denen die Präparation der V. cephalica überhaupt nicht gelingt, wurde in 2017 (noch) nicht ausgewertet. Dass der Zugangsweg über die V. subclavia durch eine höhere Rate an methodenimmanenten Komplikationen belastet ist und damit die Patientensicherheit kompromittiert, wird seit Jahren an dieser Stelle erwähnt und scheint 


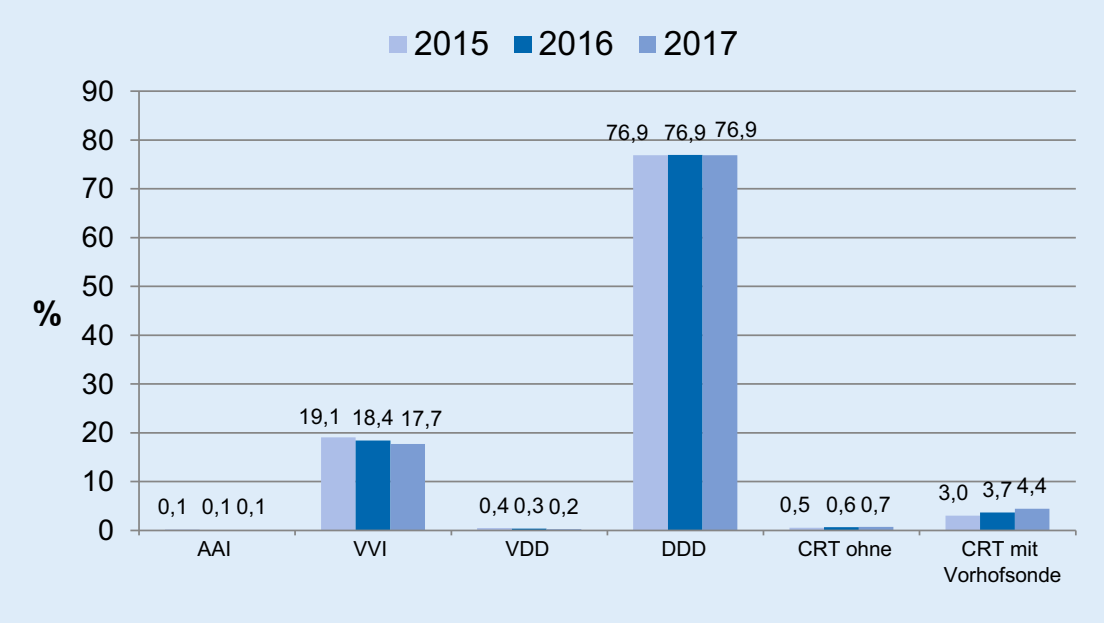

Abb. 1 A Prozentuale Verteilung der Schrittmachersysteme bei Implantation 2017 im Vergleich zu den Vorjahren

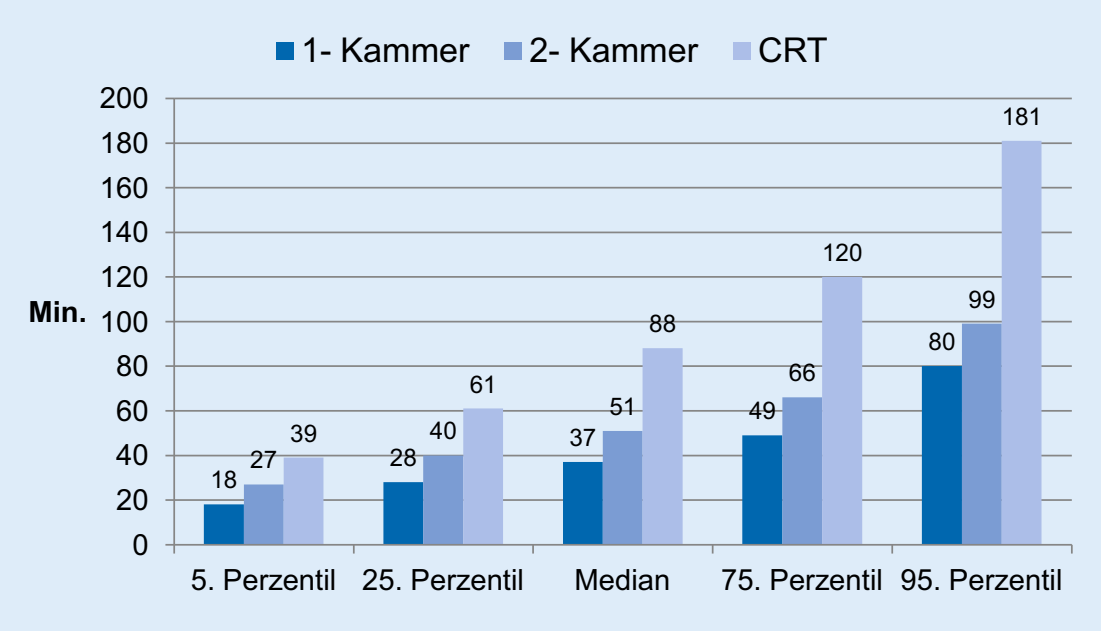

Abb. 2 A Perzentile der Operationszeiten im Jahr 2016; Beispiel: Im Jahr 2016 waren 5 \% aller 1-Kammer-Implantationen nach $18 \mathrm{~min}$ beendet

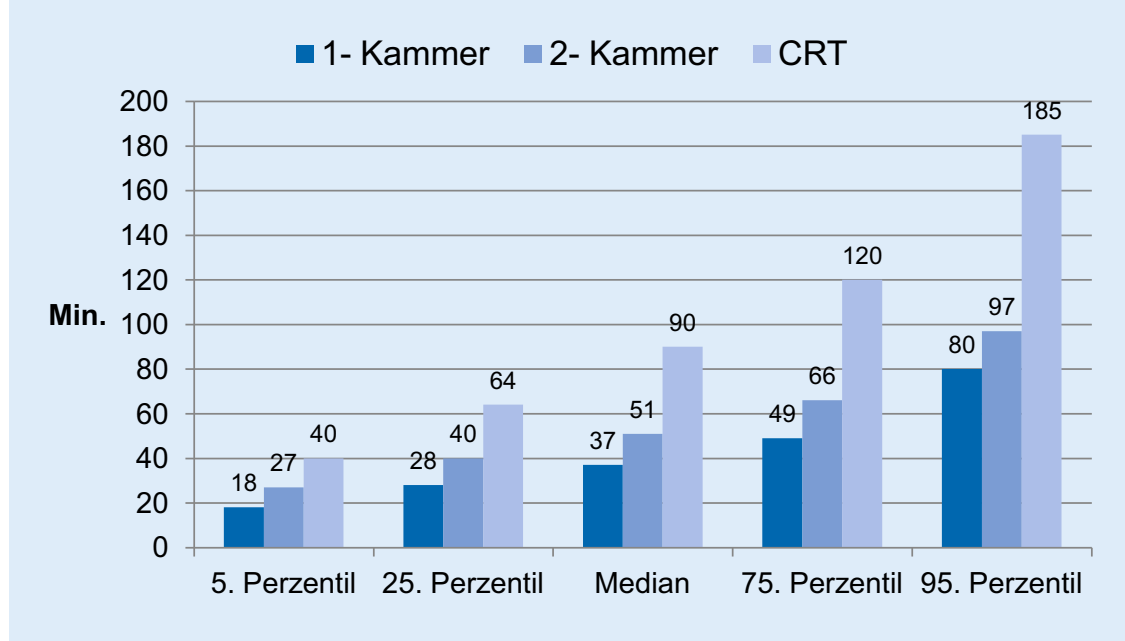

Abb. 3 A Perzentile der Operationszeiten im Jahr 2017. Beispiel: Im Jahr 2017 waren $5 \%$ aller 1-Kammer-Implantationen nach 18 min beendet als Botschaft seit 2016 angekommen $\mathrm{zu}$ sein. Ab dem Erfassungsjahr 2018 werden wir auch ohne Sonderauswertungen über detailliertere Kennzahlen zu diesem Punkt verfügen, möglicherweise ein erster Schritt zum schon lange überfälligen Qualitätsindikator „venöser Zugangsweg“.

Die Operationszeiten sind in • Tab. 8 dargestellt; sie sind im Vergleich zum Vorjahr nahezu unverändert geblieben.

Dies wird bestätigt, wenn man die Ergebnisse für die einzelnen Perzentilen der Operationszeiten aus dem Jahr 2016 (- Abb. 2) mit den Resultaten aus dem Jahr 2017 (• Abb. 3) vergleicht.

Diese für den Leser der vergangenen Registerberichte etwas ungewohnte Darstellung der Ergebnisse ist der Tatsache geschuldet, dass die Ergebnisse nach der zuvor üblichen Auswertung [2] nicht mehr angegeben werden.

Die Auswertungen des Dosisflächenprodukts sind $\bullet$ Tab. 9 zu entnehmen. Die Ergebnisse weisen darauf hin, dass die Strahlenbelastung für die Patienten abgenommen hat.

An der Konstanz der Ergebnisse für die Reizschwellenbestimmung sowie für die Ermittlung der intrakardialen Signalamplituden hat sich auch in 2017 nichts geändert (• Tab. 10). Seit 2016 werden die Ergebnisse der Amplitudenhöhe des linksventrikulären Signals nicht mehr erhoben.

Die Ergebnisse bei den perioperativen Komplikationen zeigen weiter nur wenige Veränderungen und haben insgesamt wieder leicht zugenommen (• Abb. 4; - Tab. 11).

Für die Sondendislokationen und die Sondendysfunktionen sind die Ergebnisse detaillierter in $\bullet$ Tab. 12 dargestellt. Es zeigt sich eine leichte Zunahme der Dislokationen und ein leichter Rückgang bei den Sondendysfunktionen.

Die Sonderauswertung für den $\mathrm{Zu}$ sammenhang zwischen der Gesamtkomplikationsrate und dem venösen Zugangsweg sowie zwischen Fallzahlen und Komplikationen steht bedauerlicherweise auch für das Jahr 2017 nicht zur Verfügung. 
Tab. 8 Operationszeiten in Minuten bei Implantationen 2016 und 2017 (bezogen auf alle Fälle mit gültiger Angabe zur Opertaionsdauer $>0$ )

\begin{tabular}{l|l|l|l|l} 
SM-System & $\mathbf{2 0 1 6}$ & $\mathbf{2 0 1 7}$ & MW & Median \\
& Median & $\boldsymbol{n}$ & 41,7 & 37 \\
\hline 1-Kammer & 37 & 13.721 & 55,5 & 51 \\
\hline 2-Kammer & 51 & 59.464 & 98,0 & 90 \\
CRT & 88 & 3934 & 55,2 & 50 \\
\hline Gesamt & 50 & 77.188 & & \\
\hline MW Mittelwert & & & & \\
\hline
\end{tabular}

Tab. 10 Ergebnisse der Reizschwellenmessungen und Bestimmungen der intrakardialen Signalamplituden bei Implantationen (jeweils bezogen auf alle Fälle mit gültiger Angabe)

\begin{tabular}{llllll}
$\begin{array}{l}\text { Sonde/ } \\
\text { Messung }\end{array}$ & & $\mathbf{2 0 1 6}$ & $\mathbf{2 0 1 7}$ & & \\
\hline Vorhofsonde & Reizschwelle (V) & 0,8 & 55.698 & 0,8 & 0,8 \\
& P-Wellen-Amplitude (mV) & 2,8 & 61.071 & 3,1 & 2,8 \\
\hline \multirow{2}{*}{ Ventrikel } & Reizschwelle (V) & 0,6 & 76.975 & 0,7 & 0,6 \\
(1. Sonde) & R-Wellen-Amplitude (mV) & 12,6 & 74.651 & 12,5 & 11,8 \\
\hline LV-Sonde & Reizschwelle (V) & 1 & 4017 & 1,1 & 1 \\
& R-Wellen Amplitude (mV) & k. A. & & \multicolumn{2}{c}{ Keine Angaben } \\
\hline \multirow{2}{*}{ MW Mittelwert, SD Standardabweichung } & & & & \\
\hline
\end{tabular}

Tab. 11 Perioperative Komplikationen bei Implantationen in den Jahren 2015, 2016 und 2017 im Vergleich

\begin{tabular}{|c|c|c|c|c|c|c|}
\hline \multirow[t]{2}{*}{ Perioperative Komplikationen } & \multicolumn{2}{|l|}{2015} & \multicolumn{2}{|l|}{2016} & \multicolumn{2}{|l|}{2017} \\
\hline & $n$ & $\%$ & $n$ & $\%$ & $n$ & $\%$ \\
\hline Asystolie & 97 & 0,1 & 104 & 0,1 & 105 & 0,1 \\
\hline Kammerflimmern & 33 & 0,0 & 41 & 0,1 & 24 & 0,0 \\
\hline Pneumothorax & 353 & 0,5 & 331 & 0,4 & 347 & 0,5 \\
\hline Perikarderguss & 150 & 0,2 & 148 & 0,2 & 160 & 0,2 \\
\hline Taschenhämatom & 131 & 0,2 & 100 & 0,1 & 94 & 0,1 \\
\hline Hämatothorax & 29 & 0,0 & 35 & 0,1 & 43 & 0,1 \\
\hline Wundinfektion (CDC) & 13 & 0,0 & 11 & 0,0 & 13 & 0,0 \\
\hline Sondendislokation & 977 & 1,3 & 848 & 1,1 & 922 & 1,2 \\
\hline Sondendysfunktion & 230 & 0,3 & 211 & 0,3 & 209 & 0,3 \\
\hline $\begin{array}{l}\text { Sonst. interventionspflichtige Kompli- } \\
\text { kation }\end{array}$ & 122 & 0,2 & 87 & 0,1 & 84 & 0,1 \\
\hline $\begin{array}{l}\text { Mindestens eine perioperative Komplika- } \\
\text { tion }\end{array}$ & 1961 & 2,6 & 1805 & 2,3 & 1871 & 2,4 \\
\hline
\end{tabular}

\section{Aggregatwechsel}

Die Zahl dieser Eingriffe ist im Jahr 2017 nahezu gleich geblieben (• Tab. 1). Die Laufzeit der Aggregate liegt im Median weiter bei 9 Jahren ( Abb. 5).

Die Detailgenauigkeit der Auswertungen hat auch bei den Laufzeiten nachgelassen, so werden die Unterschiede je nach Schrittmachersystem oder-Hersteller nicht mehr ausgewertet. Damit wird ein aus Sicht des Arztes und vermut-
Tab. 9 Dosisflächenprodukt 2017 im Ver-

gleich zu den Vorjahren

Dosisflächenprodukt $\left(\mathrm{cGy}^{*} \mathrm{~cm}^{2}\right)$

\begin{tabular}{l|l|l|l} 
& $\mathbf{2 0 1 5}$ & $\mathbf{2 0 1 6}$ & $\mathbf{2 0 1 7}$ \\
\hline MW & 1143 & 1097 & 887 \\
\hline Median & 496 & 450 & 395 \\
75. Perzentil & 1100 & 1000 & 893
\end{tabular}

\section{Revisionen/Systemwechsel/ Explantationen}

Die Zahl dieser Eingriffe, die im Folgenden als Revisionen zusammengefasst werden, hat im Jahr 2017 im Vergleich $\mathrm{zu}$ den Vorjahren erneut abgenommen (• Tab. 1).

Die Zahl an Revisionen bei Patienten, die zuvor am eigenen Hause operiert wurden, geht ebenfalls weiter zurück (- Tab. 14). Bei den Indikationen zur Revision werden die Schrittmacher-Aggregat-Probleme häufiger, wohingegen die Taschen- und Sondenprobleme abnehmen (• Tab. 15).

Bei der Detailbetrachtung der Schrittmacher-Aggregatprobleme zeigt sich eine Zunahme der Fehlfunktionen/ Rückrufe sowie der Zahl der Aggregataustauschoperationen im Rahmen einer Revision aus anderer Ursache (- Tab. 16). Insbesondere Ersteres bedarf der weiteren Beobachtung.

Bei den Sondenproblemen im Vorhof bleibt die Dislokation mit Abstand die häufigste Komplikation, wohingegen bei der rechtsventrikulären Sonde Reizschwellen- und/oder Wahrnehmungsprobleme die häufigste Komplikation darstellen. Zahlenmäßig relevant traten zudem Infektionen sowie Sondenbrüche oder Isolationsdefekte auf (siehe - Tab. 17 und 18) Nahezu die Hälfte der Sondenrevisionen wird innerhalb des ersten Jahres nach der vorausgehenden Operation durchgeführt, was als Ausdruck eines prozeduralen Verbesserungspotenzials gedeutet werden kann (- Tab. 19).

Bei der chirurgischen Korrektur von Sondenproblemen ( $\bullet$ Abb. 6 bzw. 7) wird weiterhin sowohl im Vorhof aber insbesondere im Ventrikel eine Neuimplantation bevorzugt.

Die Explantation funktionsloser Sonden erfolgte in 2017 vor allem aus 


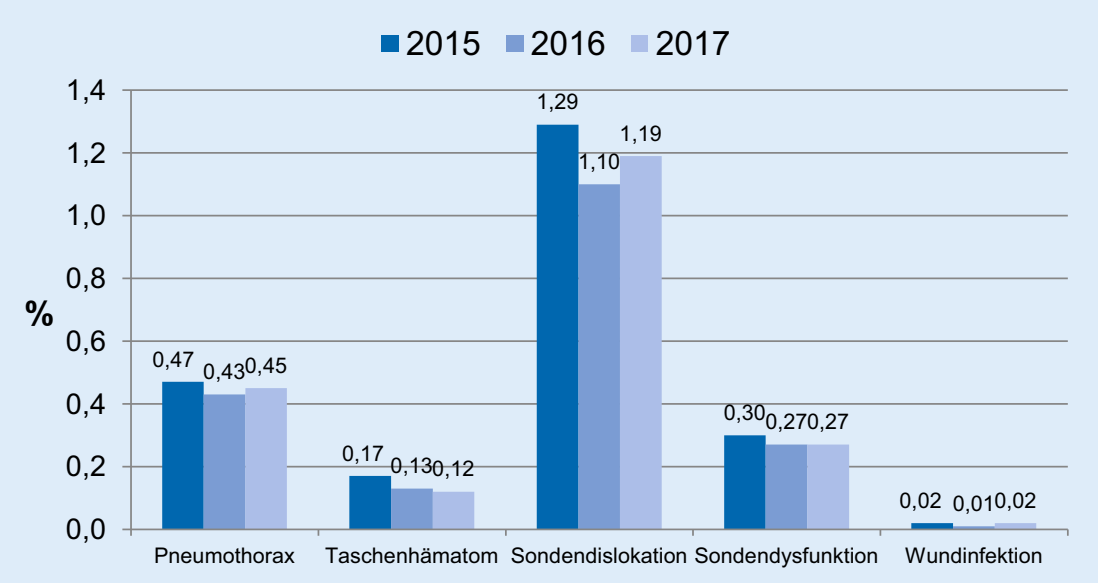

Abb. 4 ム Überblick über die perioperativen Komplikationen nach Implantation. (Sonstige: Fälle mit mind. einer der folgenden perioperativen Komplikationen: Asystolie, Kammerflimmern, interventionspflichtiger Perikarderguss, interventionspflichtiger Hämatothorax, Sondendysfunktion oder sonstige interventionspflichtige perioperative Komplikation)

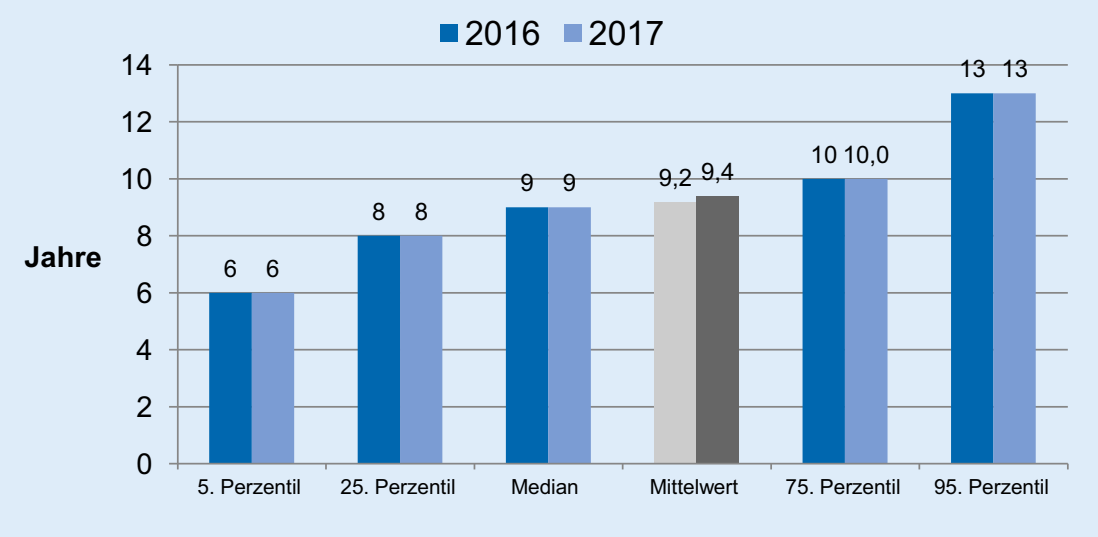

Abb. 5 A Zeit zwischen Im- und Explantation in Jahren. Beispiel: die Laufzeit eines Schrittmacheraggregats lag in $5 \%$ der Fälle in 2016 und 2017 bei $\leq 6$ Jahren

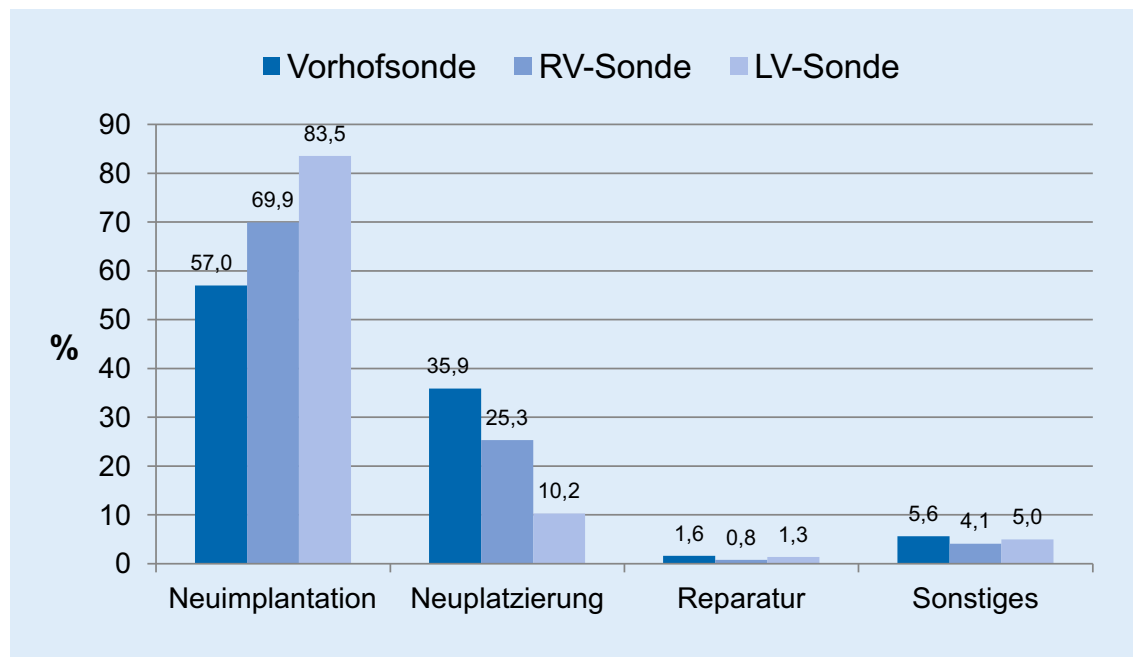

Abb. 6 \ Chirurgisches Vorgehen bei der Sondenrevision. (Bezug: Alle postoperativ funktionell aktiven Sonden, an denen ein Eingriff vorgenommen wurde) dem Koronarsinus, wohingegen im Vorhof und im rechten Ventrikel deutlich seltener explantiert wurde. Da das operative Vorgehen aber nicht nur von der Art der Sondenkomplikation, sondern auch z.B. vom zeitlichen Abstand zur vorangegangenen Operation oder den Sondeneigenschaften zum Zeitpunkt der Revision bestimmt wird, sind $\bullet$ Abb. 6 und 7 als Beschreibung und nicht als Wertung gemeint.

Die Komplikationen nach Revisionsoperationen haben an absoluter und relativer Häufigkeit erneut etwas abgenommen (•Tab. 20). Die weiterhin sehr niedrige Rate an Infektionen nach Revisionen ist sicher zum größten Teil der kurzen stationären Verweildauer geschuldet, die eine Detektion der Infektion innerhalb eines stationären Aufenthalts verhindert.

Die Sterblichkeit ist weiter nach Neuimplantationen und Revisionen am höchsten, wobei die Ursache bei den Neuimplantationen nach wie vor nicht vollständig erklärbar ist; bei einem gewissen Anteil dieser Gruppe dürfte es sich um multimorbide Patienten handeln, die akut höhergradige Bradykardien entwickeln und konsekutiv einen Herzschrittmacher erhalten, aber im weiteren Verlauf an ihrer Grunderkrankung versterben. Demgegenüber ist die Beobachtung, dass die Sterblichkeit nach Revisionen höher ist als nach Austauschoperationen verständlicher. Allerdings ist bei allen Ergebnissen zur Sterblichkeit zu bedenken, dass nicht der Tod aufgrund der Prozedur, sondern die Sterblichkeit während des stationären Aufenthalts ermittelt wird.

Im Vergleich zu den Vorjahren haben sich die Ergebnisse kaum verändert, die kontinuierliche Zunahme der Sterblichkeit nach Revisionen bedarf allerdings der weiteren Beobachtung (• Tab. 21).

\section{Kommentar mit internationalem Vergleich}

\section{Datenbasis}

Traditionsgemäß werden an dieser Stelle die Daten aus Deutschland mit anderen europäischen Registerberichten verglichen. Für das Jahr 2017 liegen wiede- 
Tab. 12 Perioperative Komplikationen bei Sonden in den Jahren 2015, 2016 und 2017 (jeweils bezogen auf alle Fälle mit dem jeweiligen Sondenproblem, z. B. im Jahr 2015 ereigneten sich 546 bzw. 55,9\% aller Sondendislokationen im Vorhof)

\begin{tabular}{|c|c|c|c|c|c|c|}
\hline \multirow[t]{2}{*}{ Sondenprobleme } & \multicolumn{2}{|c|}{2015} & \multicolumn{2}{|c|}{2016} & \multicolumn{2}{|c|}{2017} \\
\hline & $n$ & $\%$ & $n$ & $\%$ & $n$ & $\%$ \\
\hline \multicolumn{7}{|l|}{ Dislokationen } \\
\hline Vorhofsonde & 546 & 55,9 & 484 & 57,1 & 551 & 59,8 \\
\hline 1. Ventrikelsonde & 454 & 46,5 & 377 & 44,5 & 388 & 42,1 \\
\hline LV-Sonde & 8 & 0,8 & 11 & 1,3 & 14 & 1,5 \\
\hline Beide Ventrikelsonden & 0 & 0,0 & 0 & 0,0 & 0 & 0,0 \\
\hline \multicolumn{7}{|l|}{ Dysfunktionen } \\
\hline Vorhofsonde & 64 & 27,8 & 59 & 28,0 & 56 & 26,8 \\
\hline 1. Ventrikelsonde & 173 & 75,2 & 169 & 80,1 & 160 & 76,6 \\
\hline LV-Sonde & 3 & 1,3 & 3 & 0,5 & 6 & 2,9 \\
\hline Beide Ventrikelsonden & 0 & 0,0 & 0 & 0,0 & 0 & 0,0 \\
\hline
\end{tabular}

Tab. 13 Perioperative Komplikationen bei Aggregatwechseln

\begin{tabular}{|l|l|l|l|l|l|l|}
\hline Art der Komplikation & $\mathbf{2 0 1 5}$ & \multicolumn{2}{l}{$\mathbf{2 0 1 6}$} & \multicolumn{2}{l}{2017} \\
& $\boldsymbol{n}$ & $\mathbf{\%}$ & $\boldsymbol{n}$ & $\%$ & $\boldsymbol{n}$ & $\%$ \\
\hline Asystolie & 7 & 0,04 & 5 & 0,03 & 7 & 0,04 \\
\hline Kammerflimmern & 5 & 0,03 & 3 & 0,02 & 0 & 0,00 \\
\hline Taschenhämatom & 28 & 0,15 & 20 & 0,11 & 16 & 0,09 \\
\hline Wundinfektion (CDC) & 2 & 0,01 & 3 & 0,02 & $\leq 3$ & 0,01 \\
\hline Sonst. interventionspflichtige Komplikation & 13 & 0,07 & 16 & 0,09 & 10 & 0,06 \\
\hline Mindestens eine perioperative Komplikation & 49 & 0,26 & 46 & 0,26 & 34 & 0,20
\end{tabular}

Tab. 14 Ort des letzten Eingriffs, welcher der Revisionsoperation vorausging

\begin{tabular}{lllllllll}
\hline $\begin{array}{l}\text { Ort des letzten Eingriffs vor } \\
\text { der aktuellen Operation }\end{array}$ & $\mathbf{2 0 1 5}$ & $\boldsymbol{n}$ & $\mathbf{\%}$ & $\boldsymbol{n}$ & $\mathbf{2 0 1 6}$ & $\boldsymbol{n}$ & $\%$ \\
\hline Eigene Institution, stationär & 7814 & 64,0 & 7353 & 62,2 & 7083 & 62,2 \\
\hline Eigene Institution, ambulant & 89 & 0,7 & 68 & 0,6 & 79 & 0,7 \\
\hline Andere Institution, stationär & 4158 & 34,1 & 4253 & 36,0 & 4099 & 36,0 \\
\hline Andere Institution, ambulant & 148 & 1,2 & 148 & 1,3 & 122 & 1,1 \\
\hline Alle Eingriffe & 12.209 & 100 & 11.822 & 100 & 11.383 & 100
\end{tabular}

Tab. 15 Indikation zur Revisionsoperation (Mehrfachnennung möglich, ab 2015 zudem 2 neue, zusätzliche Schrittmacher-Aggregat-Probleme: vorzeitiger Aggregataustausch anlässlich einer Revisionsoperation/eines Systemwechsels und sonstige aggregatbezogene Indikation)

\begin{tabular}{lllllllll}
\hline Indikation zur Revision & $\mathbf{2 0 1 5}$ & & $\mathbf{2 0 1 6}$ & & $\mathbf{2 0 1 7}$ & \\
& $\boldsymbol{n}$ & $\mathbf{\%}$ & $\boldsymbol{n}$ & $\mathbf{\%}$ & $\boldsymbol{n}$ & $\%$ \\
\hline Schrittmacher-Aggregat-Problem & 4778 & 33,1 & 4711 & 33,7 & 5040 & 36,1 \\
\hline Taschenproblem & 1803 & 12,5 & 1702 & 12,2 & 1645 & 11,8 \\
\hline Sondenproblem & 7856 & 54,4 & 7559 & 54,1 & 7280 & 52,1
\end{tabular}

rum Berichte aus der Schweiz und Schweden vor $[3,4]$.

Die ansonsten sehr lesenswerten Berichte der European Heart Rhythm Association (EHRA; [5]) sowie der britischen Kollegen [6] aus dem Jahr 2017 sind aufgrund der nicht vollständigen Daten nicht geeignet.
(• Abb. 8). Die Zahl der Neuimplantationen pro Einrichtung ist insbesondere in Schweden deutlich höher als in der Schweiz oder in Deutschland. In allen Ländern ist inzwischen nur noch ein geringer Anstieg der Implantationsraten zu verzeichnen.

Die hohe Implantationsrate in Deutschland lässt sich seit Jahren mit den zur Verfügung stehenden Daten nicht plausibel erklären. Das Alter der Patienten bietet bei weitestgehend vergleichbarer Altersstruktur weiterhin keinen Anhalt. Im Vergleich zum Vorjahr ist der Anteil der Hochbetagten in der Schweiz wieder gesunken, in Schweden gleich geblieben und in Deutschland leicht angestiegen (• Abb. 9).

\section{EKG-Indikationen zur Schritt- machertherapie}

Bei den EKG-Indikationen (• Abb. 10) gibt es kaum Veränderungen: Der AVBlock ist weiterhin in allen 3 Ländern die häufigste Bradykardieform, und die Sinusknotenerkrankungen sind in Deutschland weiterhin vergleichsweise häufiger als in anderen Ländern eine Indikation zur Schrittmachertherapie.

\section{Schrittmachersystemauswahl}

Nachdem AAI-Systeme so gut wie gar nicht mehr implantiert werden, und auch bei VDD-Systemen mit Ausnahme der Schweiz (2,4\%) keine nennenswerten Implantationsraten mehr berichtet werden, sind in $\bullet$ Abb. 11 nur die Implantationsraten an VVI-, DDD- und CRT-Systemen aufgeführt. VVI-Systeme werden weiterhin in der Schweiz am häufigsten implantiert, DDD-Systeme in Deutschland am häufigsten verwendet, wohingegen die Rate der Implantationen von CRT-Systemen in Deutschland vergleichsweise am niedrigsten von allen 3 Ländern bleibt.

\section{Operationsdaten} bereitung für einen Vergleich weiterhin

Der Vergleich der Datenbasis zeigt das bekannte Bild: In Deutschland werden sowohl absolut (๑ Tab. 22) als auch relativ die meisten Schrittmacher implantiert
Bei der Verwendung der V. cephalica zum Sondenvorschub gibt es auch im Jahr 2017 wenig Veränderungen, nur in Schweden hat die Rate abgenommen, in der Schweiz und in Deutschland gering- 
Tab. 16 Indikation zur Revisionsoperation bei Schrittmacher-Aggregat- oder Taschenproblemen bzw. Systemumwandlungen (Mehrfachnennungen möglich)

\begin{tabular}{lllllllll} 
Schrittmacher-Aggregatproblem & $\mathbf{2 0 1 5}$ & & $\mathbf{2 0 1 6}$ & \multicolumn{2}{l}{$\mathbf{2 0 1 7}$} \\
& $\boldsymbol{n}$ & $\mathbf{\%}$ & $\boldsymbol{n}$ & $\mathbf{\%}$ & $\boldsymbol{n}$ & $\%$ \\
\hline Batterieerschöpfung & 3241 & 49,2 & 3090 & 48,2 & 2925 & 43,8 \\
\hline Fehlfunktion/Rückruf & 59 & 0,9 & 68 & 1,1 & 189 & 2,8 \\
\hline Aggregataustausch bei Revision & 730 & 11,1 & 820 & 12,8 & 1063 & 15,9 \\
\hline Anderes Aggregatproblem & 748 & 11,4 & 733 & 11,4 & 863 & 12,9 \\
\hline Taschenhämatom & 125 & 1,9 & 107 & 1,7 & 91 & 1,4 \\
\hline Infektion & 675 & 10,3 & 726 & 11,3 & 716 & 10,7 \\
\hline Aggregatperforation & 286 & 4,3 & 257 & 4,0 & 212 & 3,2 \\
\hline Anderes Taschenproblem & 717 & 10,9 & 612 & 9,5 & 626 & 9,4 \\
\hline Summe & 6581 & 100 & 6413 & 100 & 6685 & 100
\end{tabular}

Tab. 17 Indikationen zur Revisionsoperation bei Sondenproblemen

\begin{tabular}{|l|l|ll}
\hline Sondenprobleme 2017 & $\begin{array}{l}\text { Sonde } \\
\text { Vorhof }\end{array}$ & $\begin{array}{l}\text { Ventrikel } \\
\text { RV }\end{array}$ & $\begin{array}{l}\text { Ventrikel } \\
\text { LV }\end{array}$ \\
\hline Dislokation & 1398 & 998 & 63 \\
\hline Sondenbruch/Isolationsdefekt & 332 & 682 & 17 \\
\hline Fehlerhafte Konnektion & 60 & 59 & 4 \\
\hline Zwerchfell/Pectoraliszucken & 31 & 89 & 23 \\
\hline Inhibition durch Muskelpotentiale/Oversensing & 51 & 110 & - \\
\hline Wahrnehmungsfehler/Undersensing & 136 & 217 & - \\
\hline Stimulationsverlust/Reizschwellenanstieg & 406 & 1929 & 36 \\
\hline Infektion & 548 & 614 & 59 \\
\hline Myokard- oder Koronarsinusperforation & 72 & 244 & $\leq 3$ \\
\hline Sonstige & 214 & 227 & 21 \\
\hline Summe & 3248 & 5169 & 226
\end{tabular}

Tab. 18 Relative Häufigkeit der Indikationen zur Revisionsoperation bei Sondenproblemen (Prozentzahlen bezogen auf die Summe der Probleme der jeweiligen Sonde)

Sondenprobleme 2017

Sonde

Vorhof Ventrikel Ventrikel

(\%)

Dislokation

43,0

Sondenbruch/Isolationsdefekt

10,2

Fehlerhafte Konnektion

Zwerchfell/Pectoraliszucken

1,8

Inhibition durch Muskelpotentiale/Oversensing

1,0

Wahrnehmungsfehler/Undersensing

1,6

4,2

12,5

RV (\%)

19,3

LV (\%)

Stimulationsverlust/Reizschwellenanstieg

16,9

13,2

27,9

Infektion

2,2

Myokard- oder Koronarsinusperforation

Sonstige fügig zugenommen. Die Schweden verwenden weiterhin die V. cephalica deutlich häufiger als die Deutschen und diese wiederum häufiger als die Schweizer (• Tab. 23).

Bei den Operationszeiten bleiben die Schweden weiterhin - mit Ausnahme der VVI-Systeme - schneller als die Deutschen und diese wiederum schneller als die Eidgenossen. Die deutsche Auswertung unterteilt bei den 1-Kammer-Systemen nicht mehr nach AAI- und VVI, sodass die entsprechenden Zahlen nur eingeschränkt vergleichbar sind (• Abb. 12).

Die Auswahl der Vorhof- und Ventrikelsonden zeigte über die Jahre hinweg ein nahezu unverändertes Bild bzw. einen unveränderten Trend: Es wurden fast ausschließlich bipolare Sonden verwendet, die im Vorhof fast immer und im Ventrikel über die Jahre zunehmend über aktive Fixationsmechanismen verfügten, wobei in Deutschland die Vorliebe für ventrikuläre Ankerelektroden nach wie vor am größten war. Da diese Daten in Deutschland seit 2017 nicht mehr erfasst werden, ist auch kein Vergleich mit der Schweiz und Schweden möglich.

\section{Zusammenfassung und Ausblick}

Seit Jahren wurden die Registerberichte traditionell abgeschlossen durch die Betrachtung von 3 Ergebnissen, die man als Indikatoren für unterschiedliche Aspekte der Therapie mit Herzschrittmachern bewerten konnte. Die Ergebnisse waren jeweils Resultate von Sonderauswertungen durch die BQS, das AQUA-Institut oder das IQTIG. Aufgrund der ungeklärten Formalia zur sekundären Datennutzung (siehe Einleitung) müssen wir diese Daten nach 2015 und 2016 auch in 2017 schuldig bleiben, hoffen aber, dass sie irgendwann, möglicherweise schon für 2018 wieder zur Verfügung stehen werden.

Die Zusammenfassung dieses Registerberichts Herzschrittmacher schließt bei den anderen Aspekten erneut nahtlos an die Berichte der vergangenen Jahre an:

1. Die Implantationszahlen in Deutschland zählen weltweit mit zu den höchsten und haben sich auf hohem Niveau stabilisiert. 
Tab. 19 Zeitlicher Abstand der Revisionsoperation bei Sondenproblemen aller Art zum vorausgehenden Eingriff

\begin{tabular}{lll|l|l|l|l}
\hline Zeitpunkt & \multicolumn{2}{l}{ Vorhofsonde } & \multicolumn{2}{l}{ RV-Sonde } & \multicolumn{2}{l}{ LV-Sonde } \\
& $\boldsymbol{n}$ & \% & $\boldsymbol{n}$ & \% & $\boldsymbol{n}$ & $\%$ \\
\hline S1 Jahr & 2888 & 53,7 & 3029 & 50,6 & 142 & 46,9 \\
\hline >1 Jahr & 2454 & 45,6 & 2913 & 48,7 & 158 & 52,1 \\
\hline Unbekannt & 35 & 0,7 & 41 & 0,7 & 3 & 1,0
\end{tabular}

Tab. 20 Perioperative Komplikationen bei Revision, Systemumstellung, Explantation

\begin{tabular}{lll|l|l|l|l|}
\hline Art der Komplikation & $\mathbf{2 0 1 5}$ & \multicolumn{3}{l}{$\mathbf{2 0 1 6}$} & \multicolumn{2}{l}{$\mathbf{2 0 1 7}$} \\
& $\boldsymbol{n}$ & $\mathbf{\%}$ & $\boldsymbol{n}$ & $\mathbf{\%}$ & $\boldsymbol{n}$ & $\%$ \\
\hline Asystolie & 21 & 0,17 & 13 & 0,11 & 11 & 0,10 \\
\hline Kammerflimmern & 2 & 0,02 & 6 & 0,05 & 7 & 0,06 \\
\hline Pneumothorax & 31 & 0,25 & 27 & 0,23 & 34 & 0,30 \\
\hline Hämatothorax & 10 & 0,08 & 8 & 0,07 & 12 & 0,11 \\
\hline Perikarderguss & 27 & 0,22 & 15 & 0,13 & 16 & 0,14 \\
\hline Taschenhämatom & 38 & 0,31 & 30 & 0,25 & 15 & 0,13 \\
\hline Sondendislokation & 65 & 0,53 & 57 & 0,48 & 54 & 0,47 \\
\hline Sondendysfunktion & 23 & 0,19 & 26 & 0,22 & 16 & 0,14 \\
\hline Wundinfektion & 9 & 0,07 & 5 & 0,04 & 6 & 0,05 \\
\hline Sonst. Komplikation & 28 & 0,23 & 23 & 0,19 & 18 & 0,16 \\
\hline $\begin{array}{l}\text { Mindestens eine perioperative Komplika- } \\
\text { tion }\end{array}$ & 229 & 1,88 & 196 & 1,66 & 176 & 1,55
\end{tabular}

Tab. 21 Sterblichkeit im Krankenhaus bei Implantationen, Aggregatwechseln und Revisionen/ Systemwechseln/Explantationen 2017 im Vergleich zu den Ergebnissen in 2015 und 2016

\begin{tabular}{|c|c|c|c|c|c|c|}
\hline \multirow[t]{2}{*}{ Tod bei oder nach } & \multicolumn{2}{|l|}{2015} & \multicolumn{2}{|l|}{2016} & \multicolumn{2}{|l|}{2017} \\
\hline & $n$ & $\%$ & $n$ & $\%$ & $n$ & $\%$ \\
\hline Erstimplantation & 1043 & 1,08 & 1108 & 1,44 & 1144 & 1,44 \\
\hline Aggregatwechsel & 36 & 0,19 & 36 & 0,19 & 42 & 0,25 \\
\hline $\begin{array}{l}\text { Revision, Systemwechsel oder Ex- } \\
\text { plantation }\end{array}$ & 143 & 1,17 & 185 & 1,56 & 183 & 1,61 \\
\hline
\end{tabular}

\section{Tab. 22 Datenbasis im internationalen Vergleich}

\begin{tabular}{l|l|l|l}
\hline & Schweiz $^{\text {a }}$ & Schweden $^{\text {b }}$ & Deutschland $^{\mathbf{c}}$ \\
\hline Meldende Institutionen & 74 & 44 & 1099 \\
\hline Implantierende Institutionen & 74 & 44 & 1099 \\
\hline Erstimplantationen & 5246 & 7074 & 77.283 \\
\hline - Im Mittel je Institution & 71 & 161 & 70 \\
\hline - Pro 1 Mio. Einwohner & 618 & 689 & 933 \\
\hline Folgeeingriffe & 1555 & 2497 & 28.468 \\
\hline $\begin{array}{l}\text { Verhältnis Erstimplantation/Folgeeingriffe } \\
\text { Summe }\end{array}$ & 3,37 & 2,83 & 2,71 \\
\hline $\begin{array}{l}{ }^{a} \text { Einwohner in der Schweiz am 31.12.2017: 8.419.550 (Quelle: [7]) } \\
\text { bEinwohner in Schweden am 31.12.2017: 10.120.242 (Quelle: [8]) } \\
\text { 'Einwohner in Deutschland am 31.12.2017: 82.792.000 (Quelle: [9]) }\end{array}$ & & \\
\hline
\end{tabular}

2. Indikation und Systemauswahl erfolgen mit hoher Leitlinienkonformität, die in 2017 wieder die gewohnt hohen Prozentzahlen erreicht.

3. Ob Institutionen mit hohem Operationsvolumen schneller und mit weniger Komplikationen operieren, wissen wir auch in 2017 leider nicht, es wird aber wohl so geblieben sein, und

4. die Rate an Revisionsoperationen, insbesondere aufgrund von Sondenproblemen wie der Dislokation oder dem Reizschwellenanstieg, bleibt hoch, nimmt aber langsam und kontinuierlich $\mathrm{ab}$.

Zum Schluss dieses ersten Teils des Berichtes 2017 sei wieder allen, die bei der Erstellung dieses Berichts mitgeholfen haben, ganz herzlich gedankt. Weiter sei der Hoffnung Ausdruck verliehen, dass die Informationen des Berichts dem Anwender in der täglichen Praxis und damit auch den Patienten helfen. Schließlich bleibt der Wunsch, dass bei den Punkten mit Verbesserungspotenzial die Lektüre dieses Berichts dabei hilft, die Größe des Problems zu reduzieren.

Nicht unerwähnt bleiben soll der Wunsch nach einer Entscheidung in dem seit mehr als 2 Jahr bearbeiteten Verfahren der sekundären Datennutzung, damit diese Daten wieder der interessierten Öffentlichkeit zur Verfügung stehen können (s. oben).

\section{Korrespondenzadresse}

Prof. Dr. A. Markewitz, OTA a.D.

Am Goldberg 24, 56170 Bendorf, Deutschland AndreasMarkewitz@gmx.de

Mitglieder der Bundesfachgruppe Herzschrittmacher. Für die Bundesärztekammer: Prof. Dr. S. Behrens (Berlin); Prof. Dr. C. Kolb (München); Für die Deutsche Krankenhausgesellschaft: Prof. Dr. B. Lemke (Lüdenscheid); Prof. Dr. G. Fröhlig (Homburg/Saar); Für den GKV-Spitzenverband: Dr. F. Noack (Hamburg); Dr. J. van Essen (Oberursel); Für die Deutsche Gesellschaft für Kardiologie - Herz- und Kreislaufforschung: Prof. Dr. C. Stellbrink (Bielefeld); Für die Deutsche Gesellschaft für Thorax-, Herz- und Gefäßchirurgie: Dr. H. Burger, (Bad Nauheim); Für das IQTIG: Prof. Dr. A. Markewitz (Bendorf); Prof. Dr. U. Wiegand (Remscheid); Als Landesvertreter: Dr. S. Macher-Heidrich (Düsseldorf); Prof. Dr. B. Nowak (Frankfurt a. M.); Für den Deutschen Pflegerat: B. Pätzmann-Sietas (Stade); Als Patientenvertreter: M. Kollmar (Eppelheim); W.-H. Müller (Stuttgart) 


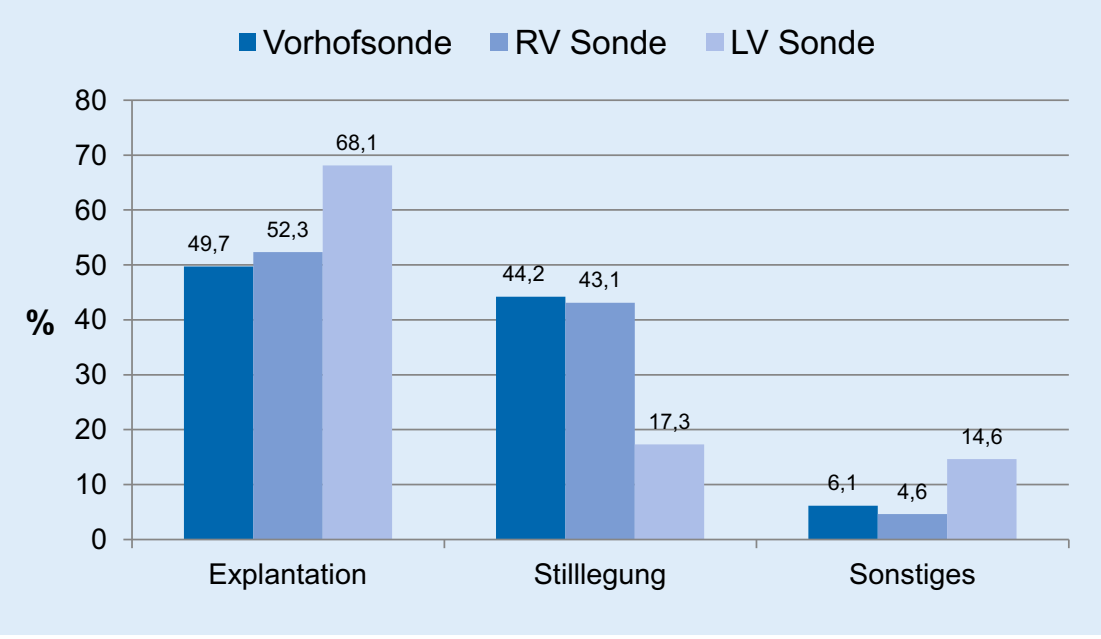

Abb. 7 A Chirurgisches Vorgehen bei funktionslosen Sonden. (Bezug: Alle postoperativ funktionell nicht aktiven Sonden bei denen die Art des Vorgehens dokumentiert wurde)

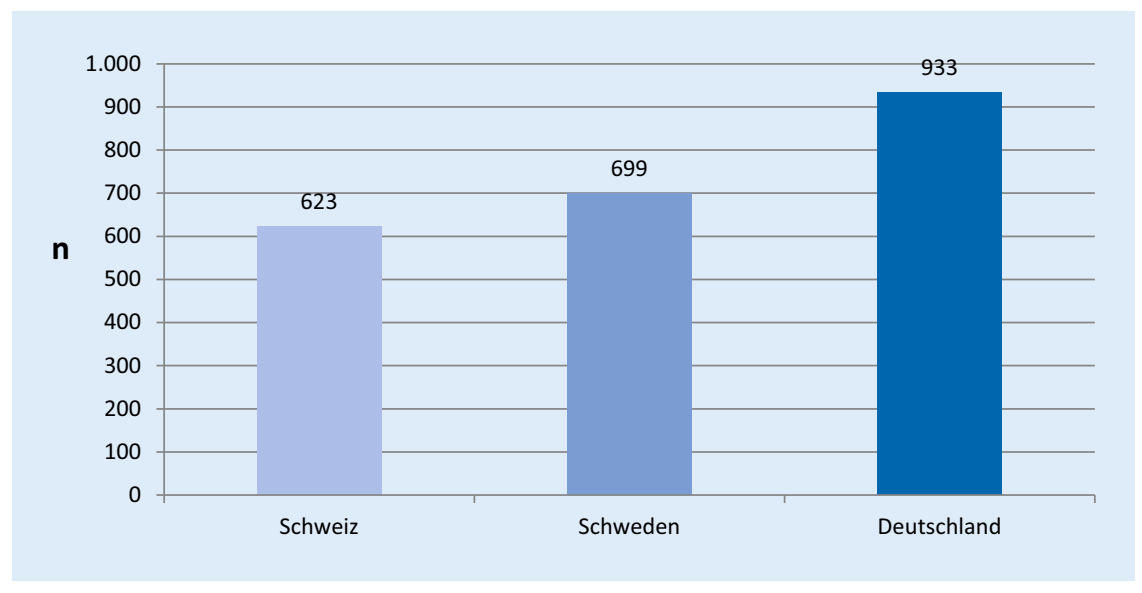

Abb. 8 ॥ Implantationen pro 1 Mio. Einwohner im internationalen Vergleich

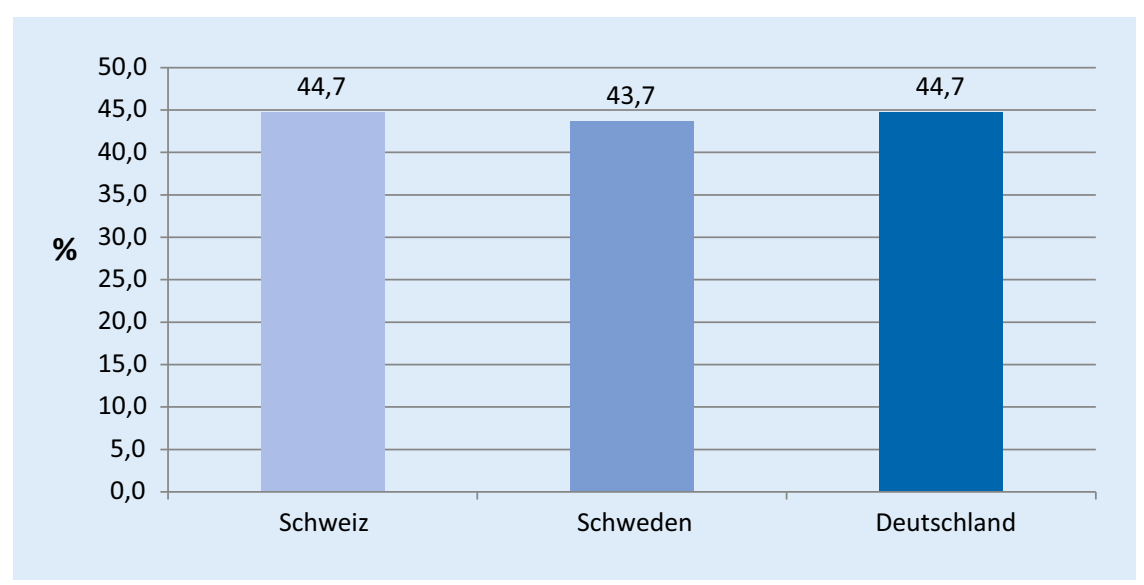

Abb. $9 \Delta$ Anteil ältererPatienten $\geq 80$ Jahre (Schweiz $\geq 81$ Jahre) an allen Patienten, bei denen ein Herzschrittmacher implantiert wurde, im Vergleich 


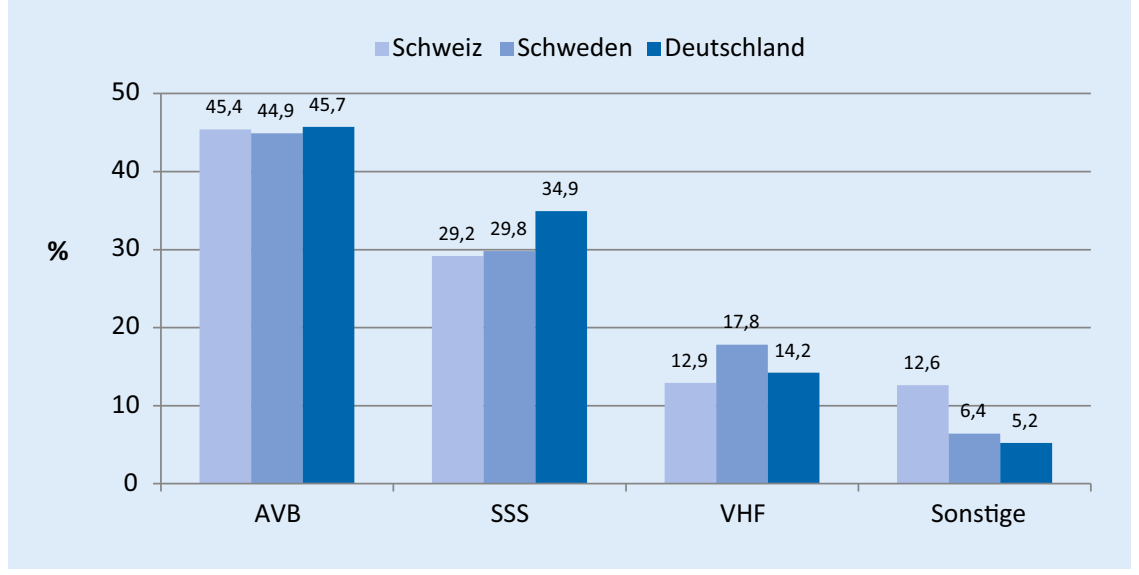

Abb. 10 ॥ EKG-Indikationen im internationalen Vergleich. (SSSSick-Sinus-Syndrom inkl. BTS, VHFbradykardes Vorhofflimmern, Sonstige Sonstige Rhythmusstörungen)

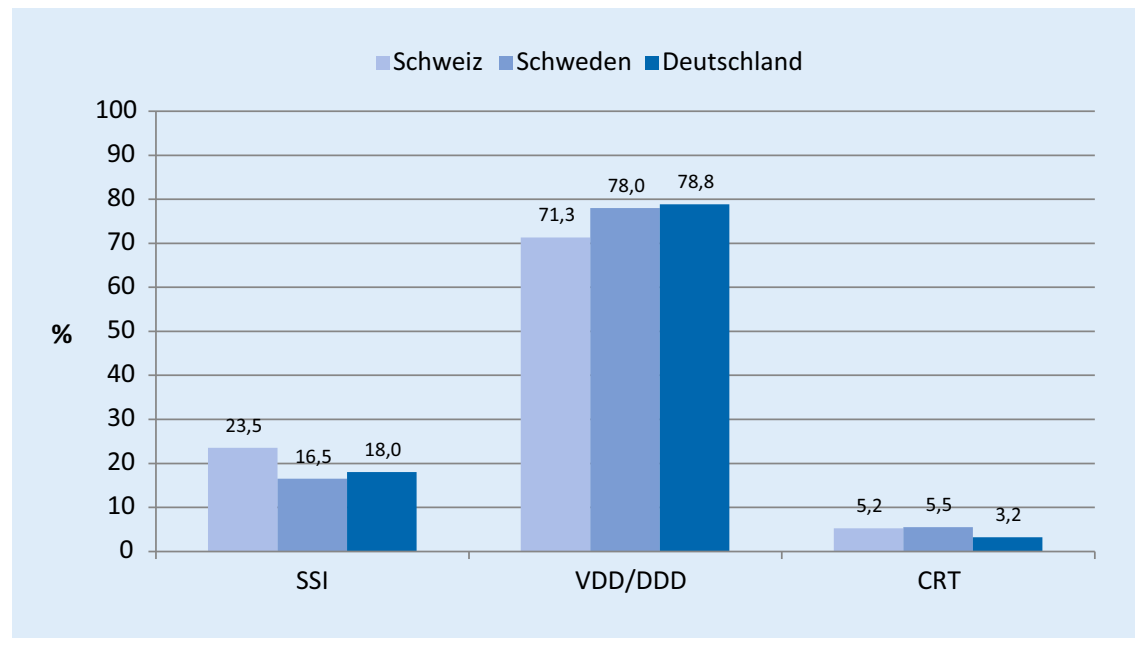

Abb. 11 A Auswahl des Schrittmachersystems im Vergleich. (SSI 1-Kammer-System [AAl oder VVI])

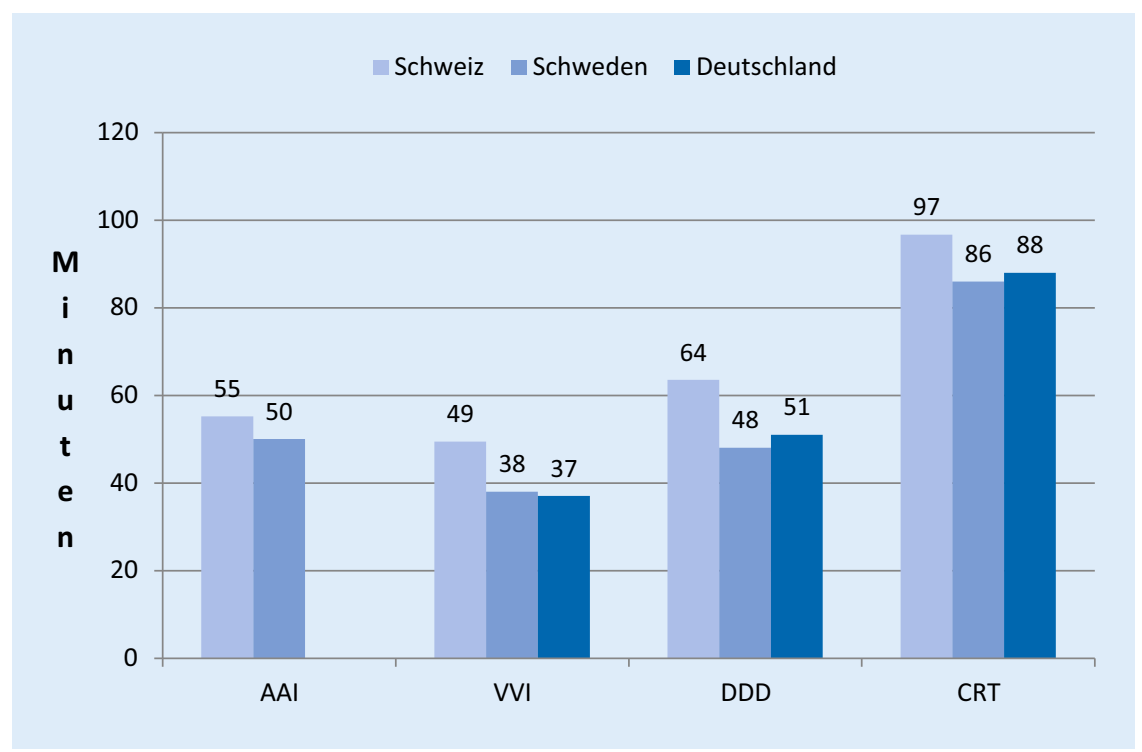

Abb. 12 a Vergleich der mittleren Operationsdauer für verschiedene Systeme (in Deutschland werden alle 1-Kammer-Systeme zusammengefasst, in der Schweiz werden VVI- und VDD-Systeme zusammengefasst) 
Tab. 23 Prozentuale Verteilung venöser Zugänge bei Neuimplantationen im Vergleich

\begin{tabular}{l|l|l|l} 
& Schweiz (\%) & Schweden (\%) & Deutschland (\%) \\
\hline V. cephalica & 29,2 & 50,1 & 37,7 \\
\hline V. subclavia & 56,8 & 37,4 & 60,2 \\
\hline Andere & 13,9 & 12,5 & 2,1
\end{tabular}

\section{Tabellenverzeichnis}

Tab. 1: Übersicht ausgewerteter Datensätze im Vergleich zum Vorjahr

Tab. 2: Operationsvolumina (Implantationen, Aggregatwechsel und Revisionen) der meldenden Krankenhäuser

Tab. 3: Vollzähligkeit der ausgewerteten Datensätze bzw. Krankenhäuser im Jahr 2016

Tab. 4: Vollzähligkeit der ausgewerteten Datensätze bzw. Krankenhäuser im Jahr 2017

Tab. 5: Demografische Daten zu Implantationen, Aggregatwechsel und Revisionen/Systemwechsel/

Explantationen

Tab. 6: führende EKG-Indikationen zur SM-Implantation in Absolutzahlen sowie in Prozent im Vergleich zum Vorjahr

Tab. 7: Venöser Zugang bei Schrittmacherimplantationen $2017 \mathrm{im}$ Vergleich zu den Vorjahren Tab. 8: Operationszeiten in Minuten bei Implantationen 2016 und 2017 (bezogen auf alle Fälle mit gültiger Angabe zur Operationsdauer $>0$ )

Tab. 9: Dosisflächenprodukt 2017 im Vergleich zu den Vorjahren

Tab. 10: Ergebnisse der Reizschwellenmessungen und Bestimmungen der intrakardialen Signalamplituden bei Implantationen (jeweils bezogen auf alle Fälle mit gültiger Angabe)

Tab. 11: Perioperative Komplikationen bei Implantationen in den Jahren 2015, 2016 und 2017 im Vergleich

Tab. 12: Perioperative Komplikationen bei Sonden 2015, 2016 und 2017 (jeweils bezogen auf alle Fälle mit dem jeweiligen Sondenproblem, z. B. im Jahr 2015 ereigneten sich 546 bzw. 55,9\% aller Sondendislokationen im Vorhof)

Tab. 13: Perioperative Komplikationen bei Aggregatwechseln

Tab. 14: Ort des letzten Eingriffs, welcher der Revisionsoperation vorausging

Tab. 15: Indikation zur Revisionsoperation (Mehrfachnennung möglich, ab 2015 zudem 2 neue, zusätzliche Schrittmacher-Aggregat-Probleme: vorzeitiger Aggregataustausch anlässlich einer Revisionsoperation/eines Systemwechsels und sonstige aggregatbezogene Indikation)

Tab. 16: Indikation zur Revisionsoperation bei Schrittmacher-Aggregat- oder Taschenproblemen bzw. Systemumwandlungen (Mehrfachnennungen möglich)

Tab. 17: Indikationen zur Revisionsoperation bei Sondenproblemen

Tab. 18: Relative Häufigkeit der Indikationen zur Revisionsoperation bei Sondenproblemen (Prozentzahlen bezogen auf die Summe der Probleme der jeweiligen Sonde)

Tab. 19: Zeitlicher Abstand der Revisionsoperation bei Sondenproblemen aller Art zum vorausgehenden Eingriff

Tab. 20: Perioperative Komplikationen bei Revision, Systemumstellung, Explantation

Tab. 21: Sterblichkeit im Krankenhaus bei Implantationen, Aggregatwechseln und Revisionen/

Systemwechseln/Explantationen 2017 im Vergleich zu den Ergebnissen in 2015 und 2016

Tab. 22: Datenbasis im internationalen Vergleich

Tab. 23: Prozentuale Verteilung venöser Zugänge bei Neuimplantationen im Vergleich

\section{Einhaltung ethischer Richtlinien}

Interessenkonflikt. A. Markewitz gibt an, dass kein Interessenkonflikt besteht.

Für diesen Beitrag wurden vom Autor keine Studien an Menschen oder Tieren durchgeführt. Für die aufgeführten Studien gelten die jeweils dort angegebenen ethischen Richtlinien.

\section{Literatur}

1. https://iqtig.org/qs-verfahren/hsm/. Zugegriffen: 4. Sept. 2019

2. https://pacemaker-register.de/. Zugegriffen: 4. Sept. 2019
3. http://www.pacemakerstiftung.ch/statistiken/ stat_2017_pm_de.pdf.Zugegriffen:5.Sept. 2019

4. https://www.pacemakerregistret.se/icdpmr/ docbank.do.Zugegriffen:5.Sept. 2019

5. https://www.escardio.org/static file/Escardio/ Subspecialty/EHRA/Publications/Documents/ 2017/ehra-white-book-2017.pdf. Zugegriffen: 16. Sept. 2019

6. https://www.nicor.org.uk/wp-content/uploads/ 2019/07/CRM-Report-2016-2017.pdf. Zugegriffen: 16. Sept. 2019

7. https://www.bfs.admin.ch/bfs/de/home/ statistiken/bevoelkerung.assetdetail.6046343. html.Zugegriffen: 17. Sept. 2019

8. http://www.scb.se/en/finding-statistics/ statistics-by-subject-area/population/populationcomposition/population-statistics/pong/tablesand-graphs/yearly-statistics--the-whole-

\section{Abbildungsverzeichnis}

Abb. 1: Prozentuale Verteilung der

Schrittmachersysteme bei Implantation 2017

im Vergleich zu den Vorjahren

Abb. 2: Perzentile der Operationszeiten im

Jahr 2016. Beispiel: Im Jahr 2016 waren $5 \%$

aller 1-Kammer-Implantationen nach $18 \mathrm{~min}$ beendet

Abb. 3: Perzentile der Operationszeiten im

Jahr 2017. Beispiel: Im Jahr 2017 waren $5 \%$

aller 1-Kammer-Implantationen nach $18 \mathrm{~min}$ beendet

Abb. 4: Überblick über die perioperativen

Komplikationen nach Implantation

Abb. 5: Zeit zwischen Im- und Explantation

in Jahren. Beispiel: die Laufzeit eines

Schrittmacheraggregats lag in 5\% der Fälle in

2016 und 2017 bei < 6 Jahren

Abb. 6: Chirurgisches Vorgehen bei der

Sondenrevision

Abb. 7: Chirurgisches Vorgehen bei

funktionslosen Sonden

Abb. 8: Implantationen pro 1 Mio. Einwohner

im internationalen Vergleich

Abb. 9: Anteil älterer Patienten $\geq 80$ Jahre

(Schweiz >81 Jahre) an allen Patienten, bei

denen ein Herzschrittmacher implantiert

wurde, im Vergleich

Abb. 10: EKG-Indikationen im internationalen Vergleich

Abb. 11: Auswahl des Schrittmacher-Systems im Vergleich

Abb. 12: Vergleich der mittleren Operationsdauer für verschiedene Systeme (in Deutschland werden alle 1-Kammer-Systeme zusammengefasst, in der Schweiz werden VVI- und VDD-Systeme zusammengefasst)

country/population-and-population-changes/. Zugegriffen: 17.Sept. 2019

9. Destatis/Stat. Bundesamt. https://www. destatis.de/DE/ZahlenFakten/GesellschaftStaat/ Bevoelkerung/Bevoelkerungsstand/Tabellen_/ Irbev03.html.Zugegriffen: 17. Sept. 2019 